\title{
Gestión de responsabilidad social universitaria en la Universidad Nacional del Centro del Perú, Huancayo-2019
}

\author{
David Raul Hurtado Tiza \\ dhurtado@uncp.edu.pe \\ Jouvet Grimanesa Flores Duran \\ yuve_22@hotmail.com \\ William Armando Reyes Alva \\ villireyes@gmail.com \\ Miguel Angel Travezaño Aldana \\ migueltravezano@gmail.com
}

\section{RESUMEN}

El objetivo general fue: Identificar la diferencia que existe entre la percepción que tienen los docentes de las facultades de Sociología y Ciencias de la Comunicación sobre el nivel de gestión de responsabilidad social universitaria en la Universidad Nacional del Centro del Perú. La metodología empleada fue cuantitativa, la tipología seguida es según lo estable Landeau (2007), básica, descriptiva, cuantitativa, transversa y de comprobación. Responde al diseño descriptivo comparativo, con una muestra censal de 40 docentes entre las facultades de CC.CC y Sociología de la UNCP, a quienes se aplicaron el cuestionario de gestión de responsabilidad social universitaria (adaptada Pro Calidad y Vallaeys), que fue validad por juicio de expertos (4) y la fiabilidad se hizo mediante prueba piloto obteniendo 0,908 según alfa de Cronbach. Se arribó la siguiente conclusión: Se identificó que existe diferencia significativa entre la percepción que tienen los docentes de las facultades de Sociología y Ciencias de la Comunicación sobre el nivel de gestión de responsabilidad social universitaria en la UNCP, Huancayo - 2019, con un nivel de significancia de $\rho_{\mathrm{c}}(0,000) \leq \rho_{\mathrm{t}}(0,05)$ y U de Mann Whitney de 393. Así mismo en sus dimensiones respectivas.

Palabras clave: RSU, Organizacional; Académico; Investigación; Social. 


\title{
Management of university social responsibility at the National University of the Center of Peru, Huancayo-2019
}

\begin{abstract}
:
The general objective was: Identify the difference that exists between the perception that teachers of the Faculties of Sociology and Communication Sciences have on the level of university social responsibility management at the National University of Central Peru. The methodology used was quantitative, the typology followed is according to Landeau (2007), basic, descriptive, quantitative, transverse and verification. It responds to the comparative descriptive design, with a census sample of 40 teachers among the faculties of CC.CC and Sociology of the UNCP, to whom the university social responsibility management questionnaire (adapted Pro Calidad and Vallaeys) was applied, which was valid by Expert judgment (4) and the reliability was done through a pilot test obtaining 0.908 according to Cronbach's alpha. The following conclusion was reached: It was identified that there is a significant difference between the perception that teachers of the Faculties of Sociology and Communication Sciences have on the level of university social responsibility management at UNCP, Huancayo - 2019, with a level of significance of $\rho \mathrm{c}$ $(0.000) \leq \rho t(0,05)$ and Mann Whitney's U of 393. Also in their respective dimensions.
\end{abstract}

Keywords: RSU, Organizational; Academic; Investigation; Social.

Artículo recibido: 17. agosto. 2020 Aceptado para publicación: 11. setiembre. 2020 Correspondencia: dahuti.epg7@gmail.com / dhurtado@uncp.edu.pe Conflictos de Interés: Ninguna que declarar 


\section{INTRODUCCIÓN}

Hoy en día, en el ambiente mundial, la responsabilidad social está posicionándose en las instituciones públicas y privadas de los diferentes sectores, enmarcando así nuevas políticas directrices, integradas y con responsabilidad económica, social y ambiental, y que estas (instituciones) deben ser conscientes de las secuelas de su accionar sobre el desarrollo sustentable que se fundamentada en el crecimiento económico para todos, con políticas sociales incluyentes y equitativas, y así fomentar una armonía entre el medio ambiente, los actores sociales e instituciones (Naciones Unidas, 2005).

Para toda, nación, institución e individuo crecer económicamente es importante, pero esto debe hacerse pensando en y para todos y todas las generaciones, donde se debe buscar asegurar las condiciones de vida de la humanidad, de la naturaleza y concordantes con los acuerdos internacionales, las políticas públicas de los estados y con participación de todos los actores sociales. Para ello, las instituciones de educación superior deben formar personas empoderadas con los asuntos de sustentabilidad, responsabilidad y ética.

Las universidades en América Latina y el Caribe, según (Universidad Construye País, 2002 ,a) se encuentran preocupados con el nuevo escenario filosófico, político, ambiental y social de gestión que va exigiendo la sociedad en pleno en cuanto a su rol, funciones y compromisos éticos, social, económico y medio ambiental, denominándose a ella responsabilidad social universitaria (RSU). Los primeros estudios sobre ella surgen en Chile bajo la premisa de buscar la percepción de RSU según los stakeholders internos de la Universidad de Telemuco y con metodologías diversas. Por su parte, (Vallaeys, 2008) menciona que en las ultimas decadas recien se estan priorizando la responsabilidad social en las univeresidades publicas y privadas de todo el mundo. Por ello, advierte que mucho de los actores sociales internos y externos vinculados con la universidad aún no conocen, comprenden ni menos asumen compromisos bajo esta nueva filosofia, politica, modelo, sistema de medicion y evaluación de la gestion de responsabilidad social univesitraia, de ahí su importancia en el presente estudio.

En el contexto peruano, el 2006 la Asamblea Nacional de Rectores (ANR), a través del Sistema Nacional de Evaluación, Acreditación y Certificación de la Calidad Educativa (SINEACE) y del Consejo de Evaluación, Acreditación y Certificación de 
la Calidad de la Educación Superior Universitaria (CONEAU), dieron el inicio al proceso de sensibilización concerniente a la autoevaluación como parte de la acreditación en las universidades públicas y privadas, bajo la mirada de mejora en la gestión de la carrera, formación académica, investigación y servicios de apoyo o extensión universitaria. Normativamente en nuestro contexto se incorporó la responsabilidad social universitaria en la Ley Universitaria $N^{\circ} 30220$, en su capítulo XIII, artículos 124 y 125, señala que la tarea de la RSU es la gestión ética y eficaz, enmarcada en la contribución y desarrollo sostenible del bienestar común de todos los actores sociales, en sus cuatro ejes de gestión, para ello la Ley indica que cada universidad debe de fijar el $2 \%$ de su presupuesto a la RSU SINEACE (2016).

Hoy en día todas las universidades tienen la obligación normativa, ético y social de considerar, implementar y ejecutar a la RSU como principio filosófico transversal de gestión en todas sus dimensiones y acciones que realice dentro y fuera de ella. Es decir, la universidad debe ser responsable de su actuar como gestor organizativo, académico, investigativo y social. También, de los impactos positivos y colaterales que producen. De la misma manera, de generar una cultura de gestión en la formación ético profesional y ciudadana de los estudiantes con valores éticos, competentes en la búsqueda de resolución de problemas sociales, ambientales y científicos de su entorno. En el ámbito local, la Universidad Nacional del Centro del Perú (UNCP), en concordancia con lo establecido por la Ley Universitaria, el Estatuto (2015) artículos 135-136, PEI (2017-2019) y demás escritos internos, indican que la gestión universitaria en sus 04 ejes tendrá la mirada con responsabilidad social. Si bien es cierto, está contemplada lo mencionado, pero ocurre que en la universidad recién se está implementando algunas acciones políticas de gestión y mejora con RS, tales como: sensibilización a autoridades de algunas áreas y facultades; reestructuración de los currículos, planes de estudio y proyección social en algunas facultades con visión y modelo de gestión de la RSU. Así mismo, la promoción de políticas institucionales orientadas al cumplimiento de los estándares de RSU en las áreas administrativas y facultades con fines de acreditación institucional y de articulación transversal en el transcurso de mejora en la gestión organizacional, academia, investigativa y participación social. 
La universidad aún tiene una agenda pendiente con la comunidad universitaria sobre la gestión de la RSU, entre ellas es la implementación de una política institucional de manera transversal en todas las áreas y estamentos, de la misma manera, considerar dentro de su estructura orgánica un área de RSU ejecutora con presupuestos acorde a ley, y el empoderamiento en toda la comunidad universitaria de lo que es RSU, porque, algunos aún creen que hablar de RSU es igual a decir proyección social o extensión universitaria o servicio a la comunidad de una manera asistencialista. Por ello, es importante saber la percepción que tienen los docentes de las facultades de Sociología y Ciencias de la Comunicación sobre la gestión de RSU, con la finalidad que la Universidad por intermedio de las facultades y específicamente los docentes empiecen a conocer, comprender y comprometerse en todo lo que involucre la RSU con su comunidad.

\section{Antecedentes del estudio:}

La investigación de (Alférez, 2014), en su investigación: La participación social curricular en la responsabilidad social universitaria: El caso de la Facultad de educación de la universidad Internacional de Catalunya. Basada bajos el enfoque gerencial, transformacional y normativo. La investigación es positivista, interpretativo y hermenéutico, con diseño cuasi experimental en un estudio de caso, con métodos, técnicas e instrumentos mixtos. La conclusión arribada fue que medir la RSU en todas sus formas son complejas ya que existen elementos invisibles que interfieren o sesgan la investigación, pero la aproximación encontrada que hay participación curricular social de forma heterogénea. Así mismo, (Alvarado, Morales y Ortíz, 2017), titulada: Un análisis de la percepción que los gerentes y profesores tienen de la RSU en las facultades de contabilidad y administración en México. Para la realización de este estudio, utilizaron un banco de base de datos digital para la A.N.FECyA.; con esa información, elaboró un modelo de ecuaciones originales que permitió analizar cómo los directivos y profesores clasifican sus percepciones sobre o RSU en sus instituciones de enseñanza. Entre sus conclusiones se indican: Estos son agentes de mayor peso en la formación y desarrollo de las competencias en los estudiantes, principalmente el fortalecimiento de sus principios, y de interpretación e interacción en la sociedad con sentido de responsabilidad. La variable organizacional, se alienta a la no corrupción, transparencia y rendición de cuentas en todos los procesos de gestión. Se observa que, 
en las carreras estudiadas, existen varios factores de gran relevancia para la percepción del RSU, entre las cuales se destacan la formación educativa y organizacional.

(Baca, 2015), en su investigación: La responsabilidad social universitaria: propuesta conceptual y medición en el ámbito de una universidad privada de Lima-Perú. La metodología empleada fue cuantitativa, de tipo no experimental, diseño descriptivo simple, muestreo probabilístico estratificado de los stakeholders, empleando la técnica de encuesta, el instrumento cuestionario de RSU y una lista de chequeo (técnica de semáforo). Las conclusiones arribadas fueron: La universidad de San Martin de Porras tiene un buen nivel de avance en RSU en las facultades de letras, pero en las facultades de ciencias aún falta alcanzar los indicadores y estándares esperados. El nivel de gestión organizacional y enseñanza son aquellos que tienen más adherencia al RSU en comparación a la de gestión ambiental, investigación y extensión social o comunal.

A nivel nacional, se tienen la investigación de (Flores, 2015), titulada: Gestión de la responsabilidad social universitaria y la calidad académica en las universidades del departamento de Puno, año 2013, en la Universidad Andina "Néstor Cáceres Velásquez". Tesis de tipo básico, descriptivo y con diseño no experimental transversal. Tuvo como muestra a 334 estudiantes a quienes aplicó cuestionario validado con el método de Kappa y una fiabilidad de 0,935 mediante alfa de Cronbach. Los resultados concerniente a RSU es que hay diferencia de apreciación delos estudiantes en la gestión de RSU en las diferentes universidades y facultades. La gestión organizacional e investigativa se encuentra en proceso y son distintas en las facultades de UNA, UANCV, UPeU, SAN CARLOS y UNAJ. Mientas, en formación académica y participación social la percepción es similar en toda la muestra de estudio. Por su parte, (Condori, 2018), en su tesis: Responsabilidad social universitaria y formación profesional en estudiantes de Psicología, 2017. Quien utilizó la metodología cuantitativa, básico, diseño no experimental, de corte transversal correlacional, con muestra de 112 estudiantes de UCV sede Ate, aplicando una encuesta estandarizada adapta por ProCalidad. Dentro de sus resultados y conclusiones se arribó: El nivel de responsabilidad social universitaria en la escuela profesional de psicología es regular y hay diferencia de opiniones respecto al nivel de las dimensiones gestión, formación e investigación, más no en proyección o extensión universitaria. 
De la misma manera, (Arispe, 2016), en su investigación: La responsabilidad social universitaria y su relación con la interculturalidad en estudiantes universitarios 2016. La metodología empleada fue cuantitativa, de tipo básico, a un nivel correlacional, con diseño transversal. La muestra fue censal de 80 estudiantes del III ciclo de la Facultad de Psicología. La técnica e instrumento empleado fue de encuesta y cuestionario respectivamente para ambas variables. Los resultados descriptivos hallados fueron: El $28,75 \%$ de los estudiantes indican que no están ni de acuerdo ni en desacuerdo con la participación social que realiza su facultad. Así mismo, se encontró que el 31,25\% de estudiantes opinaron que están en desacuerdo con el enfoque de responsabilidad social en la formación académica de su facultad. Otro, resultado es el $35 \%$ de estudiantes no están ni de acuerdo ni desacuerdo con el enfoque de responsabilidad social que se aplica en la investigación de su facultad. De la misma manera se encontró que el 38,75\% de estudiantes señalaron que no están ni de acuerdo ni desacuerdo con la gestión que se viene realizando bajo la mirada de responsabilidad social. Finalmente, en cuanto a la RSU en la facultad de Psicología los alumnos perciben que están en desacuerdo un $40 \%$. (Reyna, 2019), en su tesis titulada: Percepción de las autoridades sobre la responsabilidad social universitaria en la Universidad Nacional del Centro del Perú. Tesis Doctoral de la Universidad César Vallejo. Empleó un enfoque cualitativo, el tipo fue básico, con métodos y diseños etnográfico, fenomenológico y estudio de caso. Tuvo un muestreo cualitativo entrevistando a 9 autoridades representativas de la universidad, a quienes se hiso una entrevista a profundidad y una lista de chuequeo con la finalidad de conocer la percepción que tienen sobre la RSU. Los resultados encontrados fueron que: el $29 \%$ de los estándares si se cumplen, el $46 \%$ no se cumplen y el $25 \%$ se encuentra en proceso. La conclusión arribada es: la percepción que tienen las autoridades universitarias coinciden con la situación real de los estándares en cada categoría (gestión organizacional, formación, investigación y participación social), donde la presencia de algunos estándares tienen poco impacto y no son el resultado de un plan integral, hallándose los estándares en cada área de gestión dispersos y poco articulados, por lo que se requiere de acciones institucionales que la organicen y promuevan, con la finalidad de consolidar sistémicamente una gestión socialmente responsable con impactos internos y externos positivos. 
A nivel Regional o local: No hay investigaciones a nivel posgrado sobre la variable en estudio. Pero, si existen algunas investigaciones hechas por docentes de la UNCP, esto como parte de su función, entre ellas se tiene a (Condori, M., y Reyna, G., 2017), titulada: Percepción de la responsabilidad social universitaria en estudiantes de la Facultad de Sociologia de la U.N.C.P. La metodologia empleada fue cuantitativa, tipo basico a nivel descriptivo y con diseño no experimental transversal. La muetsra estuvo conformada por 212 estudantes, aquines se aplicaron la encuesta de percepcion de RSU de Valaleys. Teniendo como resultados respecto a la gestion organziacional y participacion social es negativa, porque no se eligen bien a las autoridades, ni estos realizan gestiones trascendentales para la universidad, tampoco cuentan con aliaznas estrategicas con otras instituciones sociales y ambientales. Mientras que, en la formación profesional e investigación la percepcion de los estudiantes es positiva, porque las asignaturas que cursan ayudan en formacion academica, cientifica y como ciudadanos responsables ante la soceidad y medio ambiente.

Otra investigación es de (Miranda, et.al., 2019) titulada: Responsabilidad social universitaria y satisfacción laboral del personal admisntrativo en una universidad pública de Huancayo (Perú). Es un estudio cuantitaivo, basico con diseño correlacional simple, con una muestra de 52 trabajadores, se emplearon dos encuestas cada una validadas con expertos y confiables. Los resultados encontrados es que hay un nivel regular de conocimiento de RSU s y existe una relacion positiva debil y significativa entre la RSU y satistaccion laboral según los trabajadores administrativo.

\section{Teoría de la pirámide}

Según, Carroll (1991) citado por (Rozas, 2009), señala que existe 4 clases de responsabilidad social que asumen las empresas como: las económicas, legales, éticas y filantrópicas, la cual le clasifico en forma jerárquica piramidal: 


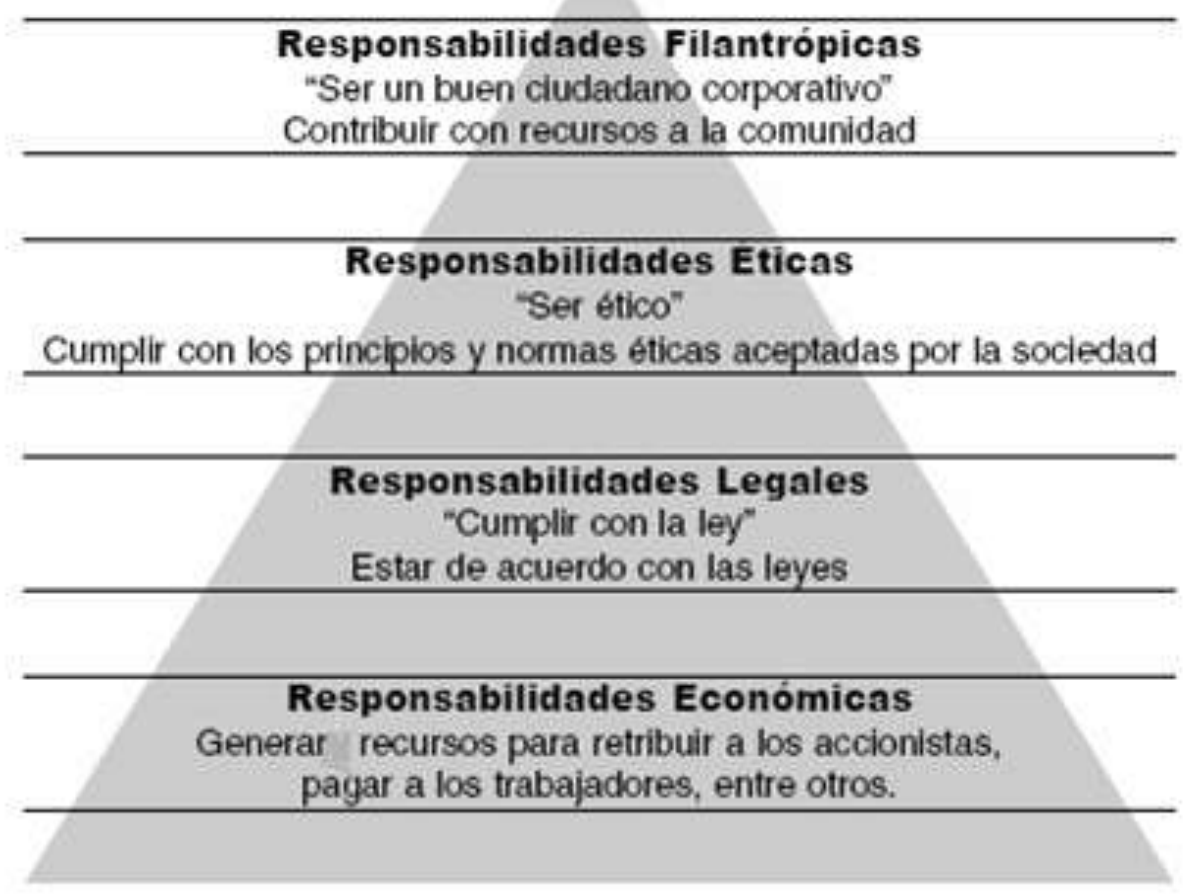

Figura 1: Teoría de la pirámide de responsabilidad social Fuente: RSE (2011) Pirámide de Carroll

http://www.manelpalencia.com/2012/02/20/responsabilidad-social-empresarial-rse-un-breve-apunte/

En la figura se aprecia que como base de la pirámide está constituida por la responsabilidad económica que significa la obtención de bienes y servicios que los usuarios requieren y anhelan, y por la que la empresa adquiere dividendos en el proceso. Mientras que las responsabilidades legales, está fundado con el cumplimento de las disposiciones y regulaciones legales. Por su parte, las responsabilidades éticas, representa el actuar de cada individuo y empresa de manera correcta, justa y equitativa ante la sociedad, menguando el perjuicio de estos. Finalmente, las responsabilidades filantrópicas buscan la calidad de vida de todos los actores sociales, para ello las empresas de forma voluntaria y corporativa deben realizar actividades y programas de promoción social.

\section{Enfoque de la responsabilidad social universitaria}

La universidad genera productos cuantitativos en función a la Responsabilidad Social en razón a los siguientes enfoques: 
El enfoque humanista: Se basa en las necesidades de los estudiantes desarrollen y promueven un bienestar propio gracias a las políticas internas de mejora constante a nivel institucional.

Sistema de enseñanza y aprendizaje: El docente aparece como protagonista, correspondiéndose con un enfoque netamente pedagógico centrándose en la investigación de los procesos educativos las cuales se manifiestan en la sociedad viendo de qué manera pueden transformar la institución educativa para ver los distintos ejes sostenibles.

Socio curricular: Está basada en la pedagogía y didáctica social como la práctica del currículo y sus diferentes componentes en los procesos formativos que están orientados al desarrollo de las habilidades y de conocimiento de los estudiantes, del personal administrativo y obviamente de los docentes. Con incidencia directa el pateamiento de estudio la educación y reflexión de la malla curricular y la aprobación de la tecnología que promueve practicas transversales de gestión educativa.

\section{Concepto de responsabilidad social universitaria (RSU)}

Para, Casani et al. (2010), La responsabilidad social universitaria está enmarcada en la interpretación que se le otorga a misión de la universidad, que implica la perspectiva de gastos de la universidad en la comunidad, que desarrolla un conjunto de actividades que "reflejan el aporte de la universidad en el entorno en que se encuentra, además de sus funciones específicas de docencia e investigación" (p. 257), que describe el valor de la responsabilidad social de la universidad en este contexto.

\section{Gestión de los impactos de la responsabilidad social en la universidad:}

Es un conjunto de acciones, decisiones y estrategias gerenciales éticamente, donde se buscan impactos sociales, humanos, sostenibles y del cumplimento de estándares por el bien común de los stakeholders en las universidades. Es decir, la gestión con RS en las universidades debe estar centradas en la trasparencia, principios, valores, confianza recíprocos, vigilancia ciudadana, alianzas estratégicas, cooperación global, innovación e investigación, con currículo sostenible en cargada de resolver problemas y de los intereses, necesidades y demandas de su entorno, a su vez de los grupos de interés y afectados. 
La gestión de responsabilidad social en las universidades, según, (Vallaeys, 2008) están directamente vinculadas con la gestión: organizacional, académica, investigación y participación social de los stakeholders, las cuales se expresan en la formación humana con ética, buscando el bien común, el cuidado del medio ambiente, desarrollo sostenible y la dinámica helicoidal entre cada uno de los impactos y la corresponsabilidad de todos los actores sociales.

\section{ORGANIZACIONAL}

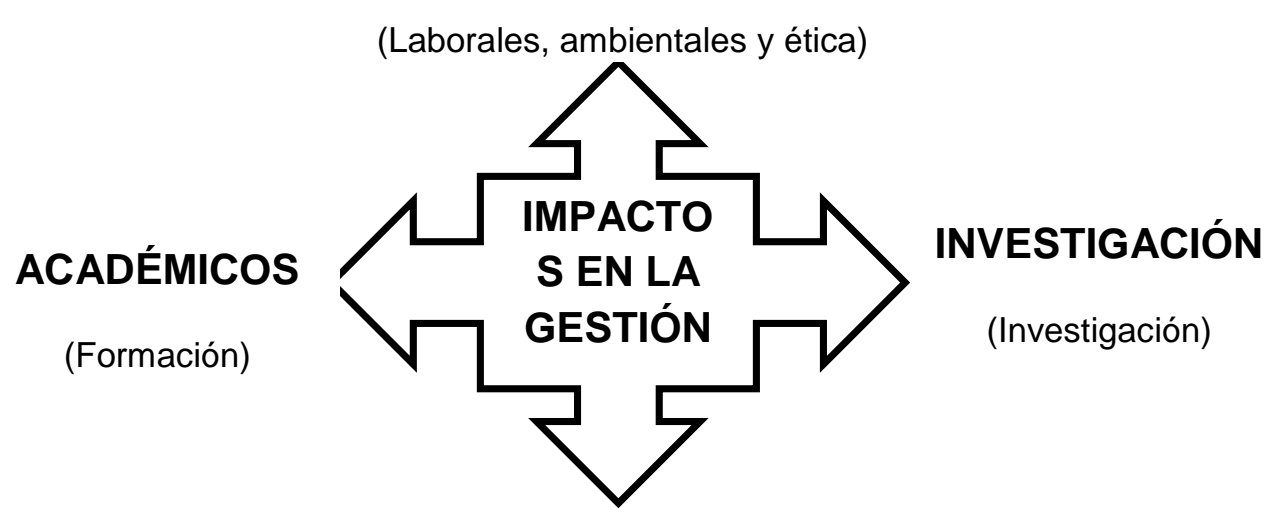

PARTICIPACIÓN SOCIAL

(Extensión y proyección social)

Figura 2: Tipos de impactos en la gestión de RS en las Universidades Fuente: Adaptada de (Vallaeys, 2008)

\section{Medición de la gestión de responsabilidad social universitaria}

Según, (Hart \& Northmore, 2010) para medir el compromiso de la universidad con la sociedad debe tener cuidado con: la diversidad de enfoques adoptados; carencia de atención de los impactos y resultados; e instrumentos y herramientas estandareizadas. Es por ello, que la medicion debe hacerse en base al impacto, gestion y cambio que realiza una universidad con la sociedad de manera responsable a toda la institución o facultades o programas. Para, la medición de la gestion de responsabilida social universitaria se establecio de manera clara y objetiva las dimensiones o categorias, indicadores y sus diferentes niveles de "entrada o recursos", "salida o productos", "resultados" e "impactos". Las dimensiones e indicadores de la gestion de responsabilidad social universitaria estan estrechamente vinculadas con las teorias, 
enfoques e impactos y sus respectivos reactivos o items, conformando el instrumento de medicón (Vallaeys, De la Cruz, 2009).

\section{Concepto de gestión de responsabilidad social universitaria}

Según, Arispe, E. (2016), considerando que la responsabilidad social universitaria permite que se fortalezca la identidad de las universidades, incurre en el cambio de una realidad social, conllevando a formar estudiantes con compromiso social de oportunidades y calidad de vida. La gestión de responsabilidad social universitaria es una organización socialmente responsable con el trabajo de articular con el compromiso de los docentes, el desarrollo de proyectos de impacto social, el voluntariado de los estudiantes; todo ello conllevará al desarrollo del país encaminado a una investigación para dar solucióm a los problemas.

Jiménez de Barros, (2001), citado por Flores, R. (2015) menciona que la gestión de responsabilidad social universitaria promueve nuevas formas de ver los problemas sociales y a la vez nuevos comportamientos para enfrentarlos, impulsa el compromiso con la sociedad de tal manera que no sólo sean simples observadores de los problemas sociales sino entes activos en la solución de las mismas. En resumen se puede decir que la gestión de RSU congregará y activará recursos materiales, económicos y humanos; capacidades, habilidades, destreza y conocimientos para poder solucionar problemas que aquejan a la sociedad y que es de interés buscar la solución y solucionarlo, todos.

\section{Dimensiones de la gestión de responsabilidad social universitaria}

Dimensión gestión organizacional: Es la política de gestión de la universidad con su comunidad, de forma democrática, imparcial, transparente, sostenible y ética, velando el clima y ambiente laboral, la gestión del talento humano, la gestión de residuos e impactos medioambientales producida por la universidad. Es decir, la universidad aplica estrategias y mecanismos de gestión para conservar el bien clima laboral, desarrollando procesos de participación de los stakeholders, en la elaboración de mestas e indicadores de desempeño, de mejora y contratación del personal democráticamente, brindando oportunidades de crecimiento profesional, respetando sus derechos laborales e integración de las diversidades en la comunidad universitaria. 
Así mismo, en campo ecológico, debe contar con una gestión integral ambiental y de residuos que la universidad produce, con la finalidad de mejorar su proceso y concientizar al personal en la cultura del cuidado del medio ambiente responsablemente, para ello debe contar con un código de ética y transparencia en las contrataciones que realice de manera óptima, eficaz, eficiente y de calidad, también debe contar con departamentos de defensoría universitaria, con políticas claras de admisión, retención, vigilancia de los estudiantes vulnerables y colaboración democrática de los stakeholders internos.

Dimensión gestión académica: La gestión académica refiere a que la universidad es la responsable de elaborar su currículo institucional con participación de los grupos de interés, bajo el rigor científico, académico, tecnológico y con directrices vigentes, atendiendo las demandas y necesidades de su entorno. La universidad y los stakeholders interno deben ser los gestores de la gestión de aprendizaje enseñanza, basadas en metodologías de ABPS, ODS y con mallas curriculares actualizadas y elaboradas por actores externos, y así ofrecer una formación integral, pertinente y que desarrolle competencias, destrezas, capacidades, habilidades blandas y actitudes proactivas de desarrollo personal, profesional y social. Es decir, el estudiante debe aprender a desaprender y viceversa, a resolver problemas de su entorno, dando alternativas de solución de manera responsabilidad, y la universidad debe ofertar y garantizar estudios sostenibles para sus egresados.

Dimensión gestión en investigación: El fin de la universidad es la investigación de los fenómenos sociales, la transferencia tecnológica y transmisión de lo que hacen y saben. La gestión investigativa es aquella que promueve los estudios inter y transdiciplinario, mediante políticas transversales en y con la comunidad. Así mismo, con convenios o alianzas estratégicas de cooperación institucional y/o con comunidades vulnerables. Los resultados de dichas investigaciones deben conducir a políticas públicas del estado.

Dimensión en gestión en participación social: Es la interacción entre todos los actores de la universidad y sociedad, en sus diferentes niveles con la promoción de proyectos de desarrollo social y colaboración recíproca. Los indicadores de la gestión en participación social están basados en la integración de la proyección social con formación e investigación, la cual buscan la articulación entre el servicio social, 
formación profesional, voluntariado estudiantil, investigación y extensión social solidaria. Par ello, los gestores deben potenciar el rol social de la universidad, haciendo trabajos en equipo entre los grupos de interés, implantando y administrando proyectos concreados, duraderos de impacto y con participación en la agenda de desarrollo local, nacional e internacional.

Problema general planteada fue: ¿Existe diferencia entre la percepción que tienen los docentes de las facultades de Sociología y Ciencias de la Comunicación sobre el nivel de gestión de responsabilidad social universitaria en la UNCP, Huancayo - 2019 ? Los problemas específicos planteadas según las 4 dimensiones son: ¿Existe diferencia entre la percepción que tienen los docentes de las facultades de Sociología y Ciencias de la Comunicación sobre el nivel de: gestión organizacional; gestión académica; gestión en investigación; y gestión en participación social en la UNCP, Huancayo - 2019?.

Hipótesis general: Existe diferencia significativa sobre la percepción que tienen los docentes de las facultades de Sociología y Ciencias de la Comunicación sobre el nivel de gestión en responsabilidad Social Universitaria en la UNCP - 2019.

Hipótesis específicas planteadas según las 4 dimensiones son: Existe diferencia significativa sobre la percepción que tienen los docentes de las facultades de Sociología y Ciencias de la Comunicación sobre el nivel de: gestión organizacional; gestión académica; gestión en investigación; y gestión en participación social en la $\mathrm{UNCP}-2019$.

Objetivo general: Identificar la diferencia que existe entre la percepción que tienen los docentes de las facultades de Sociología y Ciencias de la Comunicación sobre el nivel de gestión de responsabilidad social universitaria en la UNCP, Huancayo - 2019. Los objetivos específicos planteados según las 4 dimensiones son: Identificar la diferencia que existe entre la percepción que tienen los docentes de las facultades de Sociología y Ciencias de la Comunicación sobre el nivel de: la gestión organizacional; gestión académica; gestión en investigación; y gestión en participación social en la UNCP, Huancayo - 2019. 


\section{MATERIALES Y MÉTODOS}

La investigación se enmarcada bajo el paradigma positivista, denominada como cuantitativa, empírico-analítico y racionalista, que busca describir, explicar, predecir, descubrir y controlar los fenómenos del mundo natural, utilizando la vía hipotéticodeductivo (Tamayo, 1995). Los métodos utilizados fueron: el científico, descriptivo, deductivo, estadístico, hipotético y analítico con la finalidad de seguir la metodología cuantitativa. Así mismo, el tipo y diseño de investigación fue básica, descriptiva, cuantitativa, transversal y de comprobación (Landeau, 2007) y en concordancia a (Sierra, 2004) y (Hernández, et. al., 2014). La muestra fue censal de 40 docentes (CC.CC (18) Y Soc. (22)), se aplicaron un cuestionario de 66 preguntas relacionados a gestión de RSU, la cual fue validad por cuatro expertos y la confiablidad fue de 0,908 según alfa de Cronbach. Para, el análisis cuantitativo se utilizaron estadígrafos descriptivos e inferenciales, como las tablas de contingencia, figuras de barras y la $\mathrm{U}$ Mann Whitney y de significancia para la prueba de hipótesis. Para, la discusión de resultados se empleó el método de contrastación. Finalmente, se consideraron los aspectos éticos, ya que el quehacer de las actitudes del científico, es de buscar la verdad con el sinceramiento de información, datos, metodología científica y el compromiso de buscar las explicaciones teóricas o prácticas en la solución de problemas de su entorno. Esta actitud es prospectiva, en marcada en los lineamientos éticos de la investigación, institucional y profesional (Koepsell, 2015).

\section{RESULTADOS Y DISCUSIÓN}

En la metodología cuantitativa el método de discusión que se emplea es de contrastación, la cual está relacionada entre resultados encontrados (conclusión estadística), investigaciones realizadas (trabajos previos) y la fundamentación teórica (bases teóricas), con ellos se busca las discrepancias o concordancia entre dichos elementos, la reflexión y conclusiones parciales a la que va arribando dicha investigación (Popper, 2001) y (Tamayo, 1995).

Los resultados hallados estadísticamente, a un nivel de significancia $\rho_{\mathrm{c}}(0,000) \leq \rho_{\mathrm{t}}$ $(0,05)$ y U de Mann Whitney de 393, indican que existe diferencia significativa entre la percepción que tienen los docentes de las facultades de Sociología y Ciencias de la 
Comunicación sobre el nivel de responsabilidad social universitaria en UNCP, Huancayo - 2019. Existe investigación que contrastan dicho resultados, como el de (Alférez, 2014), (Baca, 2015) y (Condori R. , 2018), quienes encontraron que la forma como perciben los estudiantes/ administrativos y docentes varían de acuerdo a la experiencia que estas las tienen. Todos ellos emplearon metodología cuantitativa para medir la RSU. Pero, la parte contradictoria con la investigación, es que ellos emplearon un instrumento que solo mide los principios y valores de la RSU y no tanto lo que (Vallaeys F. , 2008), (Vaca, 2007) y (Flores, 2015) manifiestan que la RSU es una política filosófica que mide la gestión de RSU en su cuatro componentes o dimensiones como es la gestión organizacional, gestión académica, gestión en investigación y la gestión en participación social. Con ello, se busca que las universidades sean responsables ante su actuar con la comunidad y sus grupos de interés.

La gestión de la RSU está basada en modelos de mejora continua, gestión por y para resultados y de calidad. Los resultados encontrados no hacen más que demostrar que cada facultad tiene distintas percepciones de lo que es la RSU a nivel de sus docentes. Por su parte la facultad de Sociología, tienen una tendencia de buena a excelente gestión de RSU debido a que están más empoderados en comparación de la facultad de Ciencias de la Comunicación. Porque tienen en marcha planes de mejora con fines de acreditación de la carrera, en la que están trabajando con los grupos de interés tanto interno como externo sobre los componentes de la GRSU, referidas a clima laboral, campos ecológicos y la gestión académica e investigación.

Otro, de los resultados referidos a la gestión organizacional es que existe evidencias estadísticamente que hay diferencia significativa ente la percepción que tienen los docentes de las facultades de Sociología y Ciencias de la Comunicación sobre el nivel de gestión organizacional en la UNCP, Huancayo - 2019. Donde $\rho_{\mathrm{c}}(0,000) \leq \rho_{\mathrm{t}}(0,05)$ y U de Mann Whitney de 380. Esto significa que la gestión organizacional responsable en la Facultad de Sociología en comparación con la Facultada de Ciencias Sociales tiene mejor empoderamiento en los procesos continuos de implementación y mejora relacionados en gestión sostenible en cada componente (dimensión). La administración-organización, denominada también gestión institucional, implica el 
compromiso de las autoridades, directivos, administrativos y grupos de interés externo, mediante programas de buenas prácticas laborales y de acción ambiental.

Por su parte, el resultado comprobado estadísticamente sobre la segunda dimensión de la gestión de RSU, es que existe diferencia significativa ente la percepción que tienen los docentes de las facultades de Sociología y Ciencias de la Comunicación sobre el nivel de gestión académica en la UNCP, Huancayo -2019 . Donde $\rho_{\mathrm{c}}(0,000) \leq \rho_{\mathrm{t}}(0$, 05) y U de Mann Whitney de 396. Esto, nos conlleva a señalar que entre las facultades estudiadas esa diferenciación se basada, que uno se promueve más que en la otra la gestión de aprendizaje significativo, basado en proyectos y otros, bajo la formación profesional de este enfoque se logra intervenir en las comunidades a fin de conocer las diversas realidades que estas afrontan y poder coadyuvar en la solución de sus problemas, así se permite la formación de profesionales inmersos en el campo más que en las aulas generando una visión analítica, crítica y reflexiva; la universidad también es la encargada formar ciudadanos quienes serán los que tomen decisiones para el desarrollo de la sociedad (Vallaeys, S/F).

En cuanto al gestión en investigación, los resultados también demuestran que hay diferencia significativa $\rho_{\mathrm{c}}(0,000) \leq \rho_{\mathrm{t}}(0,05)$ y $\mathrm{U}$ de Mann Whitney de 390, entre ambas facultades. Estos resultados, reflejan que en la facultad de sociología, su currículo formativo, tiene cursos de metodología, seminarios y talleres de investigación desde el II semestre bajo la mirada del RSU, en comparación a la Facultad de Ciencias de la Comunicación que en su currícula recién llevan en los últimos semestres. Así mismo, la gestión en investigación, es un pilar y eje de las universidades y facultades, éstas deben ser responsablemente con el abordaje, proceso y publicación que coadyuve a la sociedad-empresa-universidad-ciudadano. Aquí se desarrolla la ciencia y por ende de las sociedades. Para lograr lo indicado la universidad debe promover investigaciones inter y transdisciplinarias, así mismo debe realizar convenios interinstitucionales y su labor debe tener impacto positivo en la sociedad, donde los actores que promuevan el desarrollo a través de la investigación son docentes, estudiantes y personal administrativo (Vallaeys, S/F).

Finalmente, en la gestión en participación social, entre ambas facultades se encontró que existe diferencias significativas estadísticamente con $\rho_{\mathrm{c}}(0,000) \leq \rho_{\mathrm{t}}(0,05)$ y $\mathrm{U}$ de Mann Whitney de 396. En ella, se considera a la universidad como un ente que debe 
estar vinculado con las comunidades a través de las acciones de intervención e investigación aplicada; esto se logra a través de la proyección social y extensión universitaria a favor de las comunidades más necesitadas, para lo cual la dependencia de Responsabilidad Social Universitaria a través de sus acciones debe promover la participación de docentes y estudiantes a realizar labores de proyección a las comunidades a fin de intervenir en la superación de sus problemas (Vallaeys, S/F).

\section{CONCLUSIÓN O CONSIDERACIONES FINALES}

1. Se identificó que existe diferencia significativa entre la percepción que tienen los docentes de las facultades de Sociología y Ciencias de la Comunicación sobre el nivel de gestión de responsabilidad social universitaria en la UNCP, Huancayo 2019 , con un nivel de significancia de $\rho_{\mathrm{c}}(0,000) \leq \rho_{\mathrm{t}}(0,05)$ y U de Mann Whitney de 393.

2. Se identificó que existe diferencia significativa entre la percepción que tienen los docentes de las facultades de Sociología y Ciencias de la Comunicación sobre la gestión organizacional en la UNCP, Huancayo - 2019, con un nivel de significancia $\rho_{\mathrm{c}}(0,000) \leq \rho_{\mathrm{t}}(0,05)$ y $\mathrm{U}$ de Mann Whitney de 380 .

3. Se identificó que existe diferencia significativa entre la percepción que tienen los docentes de las facultades de Sociología y Ciencias de la Comunicación sobre el nivel de gestión en académica en la UNCP, Huancayo - 2019, con un nivel de significancia de $\rho_{\mathrm{c}}(0,000) \leq \rho_{\mathrm{t}}(0,05)$ y U de Mann Whitney de 396.

4. Se identificó que existe diferencia significativa entre la percepción que tienen los docentes de las facultades de Sociología y Ciencias de la Comunicación sobre el nivel de gestión en investigación en la UNCP, Huancayo - 2019, con un nivel de significancia de $\rho_{\mathrm{c}}(0,000) \leq \rho_{\mathrm{t}}(0,05)$ y U de Mann Whitney de 390 .

5. Se identificó que existe diferencia significativa entre la percepción que tienen los docentes de las facultades de Sociología y Ciencias de la Comunicación sobre el nivel de participación social en la UNCP, Huancayo - 2019, con un nivel de significancia de $\rho_{\mathrm{c}}(0,000) \leq \rho_{\mathrm{t}}(0,05)$ y $\mathrm{U}$ de Mann Whitney de 396. 


\section{LISTA DE REFERENCIAS}

Alférez, A. (2014). Responsabilidad social universitaria en la formación curricular y la participación social: El Caso de la Facultad de Educación de la Universitat Intenacional de Catalunya. Barcelona-España: Tesis de grado. Universitat Internacional de Catalunya.

Alvarado, E. Morales, Ortiz. (Recuperado el 16 de mayo del 2019, www.redalyc,org/1872/187247578003.pdf). Un análisis sobre la percepcion que los directivos y docentes tienen de la responsabilidad social universitaria en las facultades de contaduría y adminsitración en México. Redalyc. Universidad \& Empresa. Bogota, 37-59.

Arispe, E. (2016). La responsabilidad social universitaria y su relación con la interculturalidad en estudiantes universitarios 2016. Lima - Perú: Tesis para optar grado de Maestra. Universidad San Martín de Porras. .

Baca, H. (2015). La responsabilidad social universitaria: propuesta conceptual y medición en el ámbito de una universidad privada de Lima-Perú. Sevilla España: Tesis doctoral. Universidad de Sevilla.

Condori, M. Reyna, G. (2017). Percepción de la responsabilidad social universitaria en estudiantes de la Facultad de Sociología de la U.N.C.P. Huancayo: Centro de investigación de la FS-UNCP.

Condori, R. (2018). Responsabilidad social universitaria y formación profesional en estudiantes de Psicología, 2017. Trujillo - Perú: Tesis de grado. Universidad César Vallejo.

Flores, R. (2015). Gestión de la responsabilidad social univeristaria y la calidad acdémica en las universidades del departamento de Puno año 2013. Puno - Perú: Universidad Andina Néstor Cáceres Velásquez.

Flores, P. (2015). Gestión de la responsabilidad social universitaria y la calidad académcia en las universidades del depatamento de Puno, año 2013. Puno-Perú: Tesis de grado. Universidad Andina "Nestor Cáceres Velásquez".

Hart, A. \&. Northmore. (2010). Auditoría y evaluación del compromiso entre la universidad y la comunidad: lecciones de un estudio de caso del Reyno Unido. Higher Education Quarterly, 34-58. 
Koepsell, D. (18 de Diciembre de 2015). Ética de la investigación. Obtenido de CONBIOETICA:

https://www.cooperacionib.org/libro-etica-de-la-

Investigacion-gratuito.pdf

Landeau, R. (2007). Elaboración de trabajos de investigación. Venezuela: Alfa.

Miranda, E. Hurtado, D., Fernandez, E., Victorio, D., Hurtado, O, Flores, J. (2019). Responsabilidad social universitaria y satisfaccional laboral del personal administrativo en una univerisdad pública de Huancayo (Perú). Huancayo-Perú: Centro de Investigación FS-UNCP.

Naciones Unidas. (13 de 03 de 2005). Cmisión Económica Europea. Obtenido de CEPE: http://www.unece.org/fileadmin/DAM/env/documents/2005/cep/ac.13/cep.ac.13 2005.3.rev.1.sp.dpf

Reyna, G. (2019). Percepción de las autoridades sobre la responsabilidad social universitaria en la Universidad Nacional del Centro del Perú 2018. Trujillo: Tesis Doctoral- EPG de la Universidad César Vallejo.

Rozas, A. (2009). La responsabilidad social de las empresas. Quipukamayoc. Revista de ña Facuitad de Ciencias Contables 16(32). UNMSM. Lima-Perú, 121-133.

Sierra, R. (2004). Tipos de investigación. Barcelona, España: Paraninfo S.A.

Tamayo, M. (1995). El proceso de investigación cientifica. México: Limusa S.A.

Vaca, R. (2007). Analísis de la responsabilidad social corporativa desde tres enfoques: stakeholders, capital intelectual y teoría institucional. Logroño - España: Universidad de la Rioja.

Vallaeys, F. (S/F). Breve marco teórico de la Responsabilidad social universitaria. Lima: Pontificia Universidad Católica del Perú.

Vallaeys, F. (2008). Responsabilidad social univrsitaria: una nueva filosofia de gestión ética e inteligencia para las universidades. Educación superior y sociedad, 193220.

Vallaeys, F. De La Cruz. (2009). Responsabilidad social universitaria. Manual de primeros pasos. México D.G.: McGraw-Hill. 